\title{
Numerical Analysis of Annealing Process with Local Inductive Heating on A286 Alloy Tube
}

\author{
Zhi-guo Feng*, Yang Li and Xiong-feng Liu \\ School of Mechanical Engineering, Guizhou University, Guiyang 550025, China
}

Keywords: Blind bolt, A286 Alloy, Induction Heating, Thin-walled, Local Annealing.

\begin{abstract}
Based on the local annealing process with induction heating for the A286 alloy sleeve of the blind bolt, a mathematical model is built to analyze electromagnetic-thermal coupling. Influence factors, such as frequency and current, are investigated and analyzed using finite element method on temperature distribution and length of the annealing zone in induction heating. The experiment on local annealing of the sleeve is then carried out with the optimal parameters of annealing process. Results show that temperature distribution designed in annealing zone is in a good agreement with the hardness distribution of the sleeve. The local annealing process validated by wrinkle of the sleeve under axial compression is feasible.
\end{abstract}

\section{Introduction}

Induction heating plays a key role in annealing process of the thin-walled sleeve of blind blot. Using high-frequency induction heating, the microstructure of the sleeve in local annealing zone can be softened and residual stress is released to some extent, so as to improve mechanical performance of the sleeve in local annealing zone. Therefore, controlling temperature distribution in local annealing zone is important for heat treatment of the sleeve. To obtain more accurate induction heating temperature distribution has always been one of the objectives pursued by related scholars.

Matzenmiller A and Bröcker C $[1,2]$ achieved that the cylindrical shaft made of low alloyed steel $51 \mathrm{CrV} 5$ had been soft with local inductive heating. Temperature transition in heating zone was analyzed by means of a finite element simulation. The results were essentially in agreement with experimentally measured data. Kaiser D et al. [3] proposed a novel procedure to account for high temperature gradients in the thin walled cylindrical specimens made of low alloyed steel AISI 4140 with inductive heating. The longitudinal temperature gradient and its effect on the measured length change were examined in their study. This effect was mainly caused by a strong gradient of the induced heat due to the longitudinal end effect. Yi $\mathrm{H}$ et al. [4] utilized a computing method to simulate the heat treatment process with induction heating for longitudinally-welded steel pipe and analyze electromagnetic and temperature field. This study offered quantitative reference and analysis method for investigating formation mechanism of the "double ellipsoidal" temperature field during steel pipe induction heating.

In the following parts, the annealing process on the thin-walled sleeve of the blind blot is described. To deal with the coupled electromagnetic-thermal problem, the finite element method is applied to investigate temperature field distribution. The main purpose of this work is to optimize technics parameter with an aim of drawing a conclusion about mechanical performance of local annealing zone by means of temperature transition diagrams.

\section{Numerical Model of Induction Heating}

The sleeve with a wall thickness of $0.5 \mathrm{~mm}$ has been manufactured using A286 alloy. The geometry of the hollow sleeve is depicted in Fig.1. The length of annealing zone is $8 \mathrm{~mm}$. To investigate the influence of temperature in annealing zone, the experiments are performed with induction heating, see Fig.2 (a). Taking into account the symmetry of revolution, the model domain can be simplified as the one shown in Fig.2 (b). The auxiliary device is the A286 alloy cylinder of variable diameter placed in the middle of a symmetric induction coil, which became the heat source with temperature gradient to soften the sleeve in annealing zone by means of thermal radiation. The 
distance is $0.15 \mathrm{~mm}$ between outer circle of the sleeve and inner circle the auxiliary device.

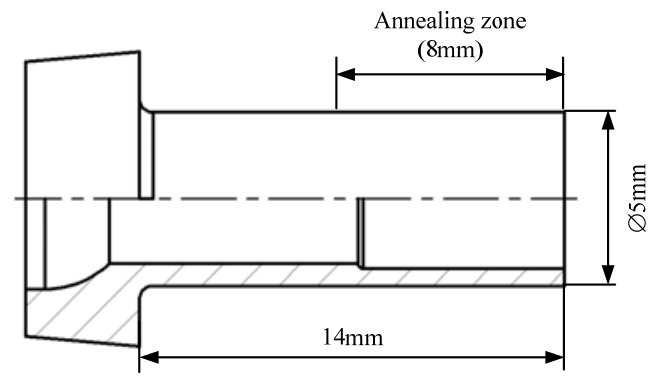

Figure 1 Geometry of the sleeve used in the experiments
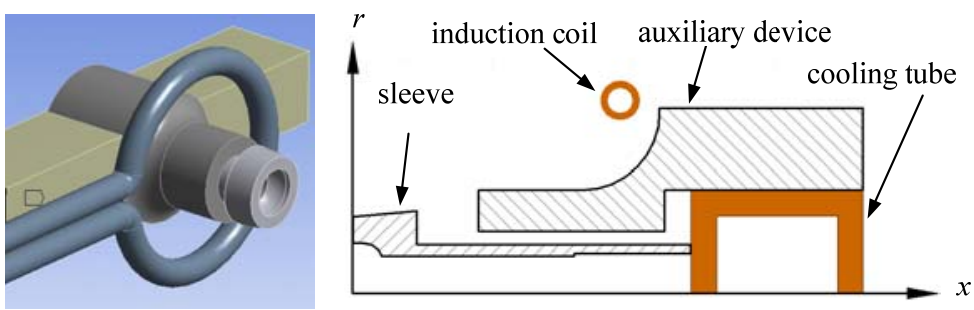

Figure 2 Schematic diagram of the sleeve annealing process in local area

\section{Material Properties}

Table 1 Chemical composition of A286 alloy (mass fraction, \%)

\begin{tabular}{ccccccccccc}
\hline $\mathrm{C}$ & $\mathrm{Mn}$ & $\mathrm{Si}$ & $\mathrm{Ni}$ & $\mathrm{Cr}$ & $\mathrm{Mo}$ & $\mathrm{V}$ & $\mathrm{Ti}$ & $\mathrm{Al}$ & $\mathrm{B}$ & $\mathrm{Fe}$ \\
\hline 0.04 & 0.20 & 0.20 & 25 & 14.5 & 1.25 & 0.30 & 2.10 & 0.15 & 0.006 & Bal \\
\hline
\end{tabular}
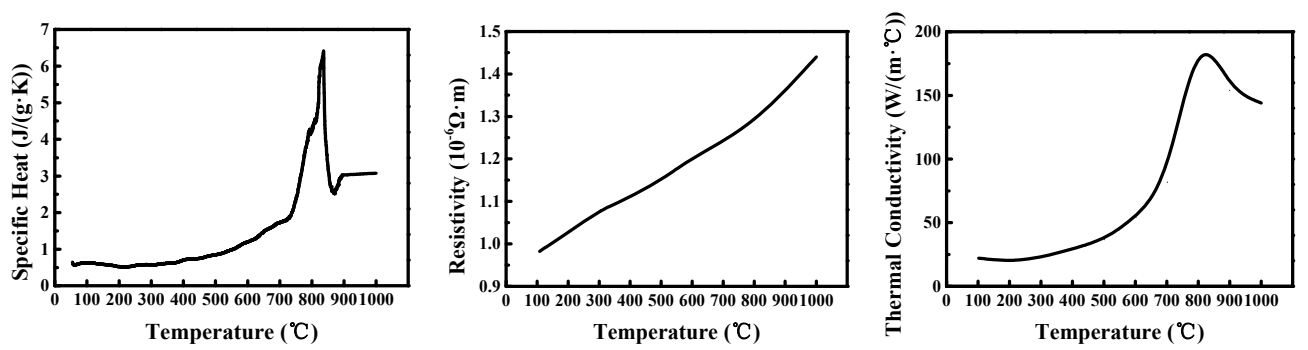

Figure 3 Temperature-dependent material properties of A286 alloy. Values for the specific heat, resistivity, and thermal conductivity are shown.

The A286 alloy sleeve is used to perform the heat experiences. The chemical composition of the A286 alloy sleeve is shown in Table 1[5]. Fig.3 shows respectively the measured specific heat capacity, resistivity and thermal conductivity of the A286 alloy.

\section{Mathematical Model}

Mathematical equations describing the electromagnetic fields are based on the following Maxwell's [6, 9]: 


$$
\begin{gathered}
\nabla \cdot \boldsymbol{B}=0 \\
\nabla \cdot \boldsymbol{D}=\alpha \\
\nabla \times \boldsymbol{E}=-\frac{\partial \boldsymbol{B}}{\partial t} \\
\nabla \times \boldsymbol{H}=\boldsymbol{J}+\frac{\partial \boldsymbol{D}}{\partial t}
\end{gathered}
$$

Where $\mathrm{B}$ is the magnetic flux density, $\mathrm{D}$ is the electric flux density, $\alpha$ is the electric charge density. $\mathrm{H}$ is the magnetic field intensity. $\mathrm{J}$ is conduction current density.

In our case, considering magneto-quasi-static problems, the displacement current density $\partial \mathrm{D} / \partial \mathrm{t}$ is negligible compared to the conduction current density. Thus, the above equations take the form:

$$
\begin{gathered}
\nabla \cdot \boldsymbol{B}=0 \\
\nabla \times \boldsymbol{E}=-\frac{\partial \boldsymbol{B}}{\partial t} \\
\nabla \times \boldsymbol{H}=\boldsymbol{J}
\end{gathered}
$$

The above field equations are supplemented by constitutive relations:

$$
\begin{array}{r}
\boldsymbol{J}=\sigma \boldsymbol{E} \\
\boldsymbol{B}=\mu \boldsymbol{H}
\end{array}
$$

Where $\sigma$ is the electric conductivity, $\mu$ is the magnetic permeability. Both $\sigma$ and $\mu$ are assumed to be isotropic and with temperature dependence.

For the solution of magnetic field problems, the magnetic potential vector A and the scalar electric potential $\varphi$ are used. The relationship between magnetic and electric potential is introduced:

$$
\begin{gathered}
\boldsymbol{B}=\nabla \times \boldsymbol{A} \\
\boldsymbol{E}=-\frac{\partial \boldsymbol{A}}{\partial t}-\nabla \frac{\partial \varphi}{\partial t}
\end{gathered}
$$

Substituting Eq.11 in Eq. 8 yields 


$$
\boldsymbol{J}=-\sigma\left(\frac{\partial \boldsymbol{A}}{\partial t}+\nabla \frac{\partial \varphi}{\partial t}\right)+\boldsymbol{J}_{s}
$$

Where Js denotes given source current density.

The Ampere's law (7) remains to be solved in conjunction with the constitutive relations:

$$
\nabla \times \frac{1}{\mu_{0} \mu_{r}} \nabla \times \boldsymbol{A}+\sigma\left(\frac{\partial \boldsymbol{A}}{\partial t}+\nabla \frac{\partial \varphi}{\partial t}\right)=\boldsymbol{J}_{s}
$$

For the uniqueness of $\mathrm{A}$, the penalty function is used employing the Coulomb gauge:

$$
-\nabla \frac{1}{\mu_{0} \mu_{r}}(\nabla \cdot \boldsymbol{A})=0
$$

Substituting Eq.14 in Eq.7 yields

$$
\nabla \times \frac{1}{\mu_{0} \mu_{r}} \nabla \times \boldsymbol{A}-\nabla \frac{1}{\mu_{0} \mu_{r}}(\nabla \cdot \boldsymbol{A})+\sigma\left(\frac{\partial \boldsymbol{A}}{\partial t}+\nabla \frac{\partial \varphi}{\partial t}\right)=\boldsymbol{J}_{s}
$$

Additionally, the divergence-free property of source current density:

$$
\nabla \cdot \boldsymbol{J}=0
$$

In permeable conducting region, corresponding to the sleeve tube, Js is assumed to be zero. The differential equations can be written as follows:

$$
\begin{gathered}
\boldsymbol{J}=-\sigma\left(\frac{\partial \boldsymbol{A}}{\partial t}+\nabla \frac{\partial \varphi}{\partial t}\right) \\
\nabla \times \frac{1}{\mu_{0} \mu_{r}} \nabla \times \boldsymbol{A}-\nabla \frac{1}{\mu_{0} \mu_{r}}(\nabla \cdot \boldsymbol{A})+\sigma\left(\frac{\partial \boldsymbol{A}}{\partial t}+\nabla \frac{\partial \varphi}{\partial t}\right)=0 \\
\nabla \cdot\left(-\sigma \frac{\partial \boldsymbol{A}}{\partial t}-\sigma \nabla \frac{\partial \varphi}{\partial t}\right)=0
\end{gathered}
$$

Eddy currents derived from the electromagnetic model manifest themselves through heat production due to the Joule effect. The heat is then distributed throughout the sleeve tube. The process is described by a classical heat equation

$$
\rho C \frac{\partial T}{\partial t}-\nabla \cdot(k \nabla T)=0
$$


Where $\rho C$ is the volumetric specific heat, $k$ is the thermal conductivity. The heat source $Q$ is caused by eddy currents, which can be calculated as $[10,11]$ :

$$
Q=\sigma|\boldsymbol{E}|^{2}
$$

The following radiation and convection boundary condition are prescribed on the sleeve's surface:

$$
k \nabla T \cdot \widehat{\mathbf{n}}+h\left(T-T_{a m b}\right)+\varepsilon \sigma_{s b}\left(T^{4}-T_{a m b}^{4}\right)=0
$$

Where $\mathrm{h}$ is the convection coefficient, Tamb is the room temperature, $\varepsilon$ is the material emissivity and $\sigma \mathrm{sb}$ is the Stefan-Boltzmann constant.

\section{Numerical Solution}

The governing equations introduced above are solved numerically using the finite element method. To carry out different stages of the numerical simulation, the following software packages, ANSOFT/Maxwell and ANSYS/Transient Thermal, are used to solve the coupled electromagnetic-thermal problem. The model domain which is shown in Fig.2 is meshed with solid186 hexahedron elements for the sleeve, auxiliary device and cooling tube. The coil is meshed with solid187 tetrahedron elements. A total of 35,221 hexahedron/tetrahedron elements and 82,448 nodes are used in our calculations. The section circle of induction coil has an outer and inner radius of $3 \mathrm{~mm}$ and $1 \mathrm{~mm}$, respectively.

\section{Results and Discussion}

Fig.4 shows distribution of magnetic field and temperature distribution of sleeve under different frequencies but the same current. In simulation, frequencies and current are respectively $50 \mathrm{kHz}, 75$ $\mathrm{kHz}, 100 \mathrm{kHz}$ and 500A. Based on frequencies, the skin depths of induction current are $1.12 \mathrm{~mm}$, $0.91 \mathrm{~mm}$ and $0.79 \mathrm{~mm}$. The maximum magnetic flux density is near the auxiliary device, which increases with frequency. Simultaneously, loss of auxiliary device in induction heating becomes bigger to produce more heat. The results of temperature distribution in annealing zone show the differences on length of annealing zone and high temperature area can be neglected. The maximum temperature on high temperature area under different frequency are respectively $716.82^{\circ} \mathrm{C}$, $876.33^{\circ} \mathrm{C}$ and $1024.20^{\circ} \mathrm{C}$. Thus the annealing temperature in induction heating shall be amplified with increased frequency.

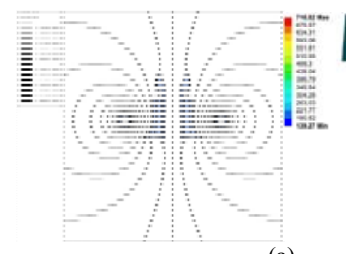

(a)

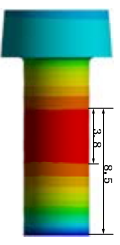

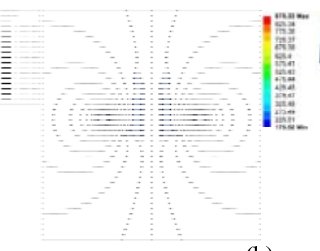

(b)

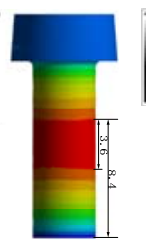

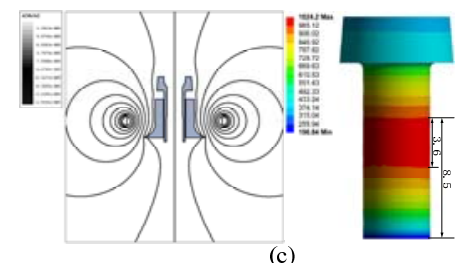

(c)

Figure 4 Distribution of magnetic field and temperature distribution of sleeve in different frequency.

(a) $f=50 \mathrm{kHz}$ (b) $f=75 \mathrm{kHz}$ (c) $f=100 \mathrm{kHz}$ 
Distribution of magnetic field and temperature distribution of sleeve under different currents are shown in Fig.5. During simulation, the currents and the frequency are respectively 400A, 500A, $600 \mathrm{~A}$ and $100 \mathrm{kHz}$. The maximum magnetic flux densities near the auxiliary device are respectively $0.062 \mathrm{~T}, 0.076 \mathrm{~T}$ and $0.082 \mathrm{~T}$, which show that there is not much difference. But the loss of auxiliary device in induction heating becomes bigger with current increased to produce more heat. The length on annealing zone is $8.6 \mathrm{~mm}, 8.5 \mathrm{~mm}$ and $8.3 \mathrm{~mm}$, respectively. The length on high temperature area is $4.9 \mathrm{~mm}, 3.6 \mathrm{~mm}$ and $3.3 \mathrm{~mm}$. The maximum temperature on high temperature area under different frequencies is $704.94^{\circ} \mathrm{C}$ and $1024.20^{\circ} \mathrm{C}$ and $1353.10^{\circ} \mathrm{C}$ respectively. The length on local annealing zone and high temperature area decreases with current increase. At the same time, the temperature in induction heating increases. Based on the above results, parameters on annealing process of the sleeve can be drawn up, see Table 2 .

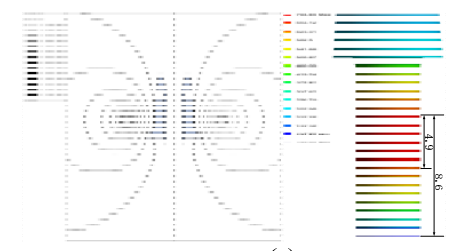

(a)

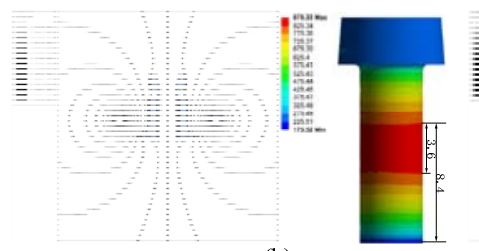

(b)

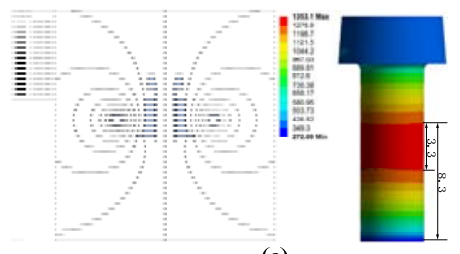

(c)

Figure 5 Distribution of magnetic field and temperature distribution of sleeve in different current.

(a) $I=400$ A (b) $I=500$ A (c) $I=600$ A

Table 2 Annealing process parameters

\begin{tabular}{llll}
\hline \multicolumn{1}{c}{ Name } & \multicolumn{2}{c}{ Name } \\
\hline Coil cross-section structure & Circular & Sectional size of coil & $\varnothing 3 \mathrm{~mm}$ \\
Inner diameter of coil & $\varnothing 20 \mathrm{~mm}$ & Electric current & $500 \sim 600 \mathrm{~A}$ \\
Frequency & $75 \sim 100 \mathrm{kHz}$ & Heating time & $15 \sim 20 \mathrm{~s}$ \\
Heating temperature(Max) & $1000 \sim 1100^{\circ} \mathrm{C}$ & Sleeve size & $\varnothing 5 \times 14 \mathrm{~mm}$ \\
\hline
\end{tabular}

\section{Induction Heating Experiment}

Fig. 6 shows how annealing equipment with induction heating is designed for heat treatment of the sleeve. During the induction heating process, the temperature in local annealing zone could be measured by an infrared thermometry (CIT-S, China). The maximum temperature was $1080^{\circ} \mathrm{C}$ in local annealing zone. The three most important values (the voltage amplitude, the current amplitude and the frequency, respectively) measured by current transformer and oscilloscope read: $U=100 \mathrm{~V}$, $I=583 \mathrm{~A}$ and $f=75 \mathrm{kHz}$. Hardness of the sleeve in annealing zone was measured with micro hardness tester (MHV-1000, China ). Fig.7 shows the hardness measured of sample I processed by our team and sample II made by Cherry Company (USA) in local annealing zone. The length of annealing zone is both $8 \mathrm{~mm}$. Hardness transformation of sample I in annealing zone is essentially in agreement with that of sample II. Ranging from $0 \mathrm{~mm}$ to $7 \mathrm{~mm}$, average hardness value of sample I is $15 \mathrm{HV}$ higher than that of sampleП. These results indicate that inductor shall be improved to adjust temperature gradient and temperature distribution in annealing zone. 


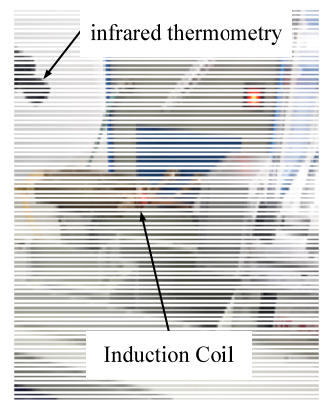

Figure 6. Annealing equipment

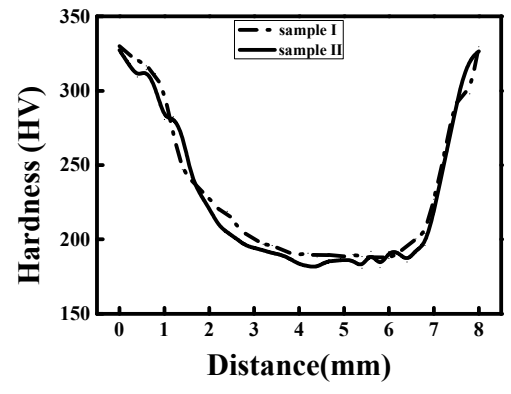

Figure 7. Hardness of the sleeve in annealing zone

Wrinkling instability in axial compression of the sleeve is shown in Fig.8. The Fig.8 (a) shows that the thickness of workpiece is $0.9 \mathrm{~mm}$, diameter of wrinkle is $6.56 \mathrm{~mm}$ and the unreformed length of the sleeve in annealing zone is $2.24 \mathrm{~mm}$. Contact between wrinkle and the upper plan of workpiece is uniformity. The Fig.8 (b) shows that the thickness of workpiece is $1.1 \mathrm{~mm}$, diameter of wrinkle is $6.60 \mathrm{~mm}$ and the unreformed width of the sleeve in annealing zone is $1.08 \mathrm{~mm}$. Contact between wrinkle and the upper plan of workpiece is satisfying. The results indicate the length of annealing zone can satisfy different thickness of workpiece and plasticity of the sleeve in annealing zone is enhanced. Future studies in this field are suggested to focus on the relation between temperature and properties of A286 alloy in annealing zone.

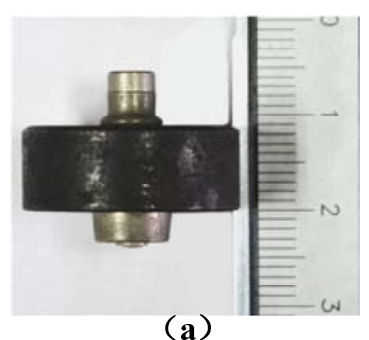

(a)

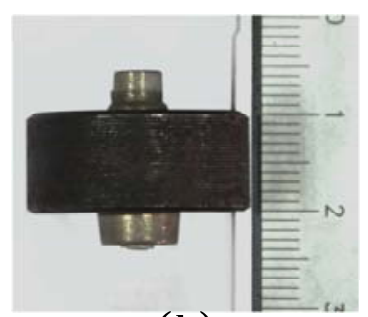

(b)

Figure 8. Wrinkle of the sleeve for different thickness of workpiece

\section{Conclusion}

This paper makes a study of the annealing process with induction heating of the A286 alloy sleeve from both the numerical and experimental way, in which the coupled electromagnetic thermal modelling of the induction heating process using the finite element method is presented, with the influence of current and frequency on annealing temperature analyzed. Based on simulation results, induction heating experiments are carried out, which results reveal an agreement with the numerical analysis on the annealing temperature distribution. In addition, the local annealing process validated by the hardness distribution and wrinkle of the sleeve in annealing zone proves feasible.

\section{Acknowledgement}

This research was financially supported by the Project on Science and Technology of Guizhou province (Grant No. GY [2012]3042) 


\section{References}

[1] A Matzenmiller, C Bröcker. Thermo-mechanically coupled FE analysis and sensitivity study of simultaneous hot/cold forging process with local inductive heating and cooling, International Journal of Material Forming. 5 (2012) 275-300.

[2] T Wagner, C Bröcker, N Saba, et al. Analysis of a thermomechanical coupled forming process using enhanced design and analysis of computer experiments, Advances in Statistical Analysis. 94 (2010) 389-404.

[3] Kaiser D, Dietrich S, Schulze V, et al. A novel procedure to account for high temperature gradients in an induction dilatometer sample during rapid heating, Thermochimica Acta. 646 (2016) 8-15.

[4] H Yi, Y Enlin, Z Tianxu. Three-dimensional analysis of medium-frequency induction heating of steel pipes subject to motion factor, International Journal of Heat and Mass Transfer. 101 (2016) 452-460.

[5] http://www.ATImetals.com.

[6] N. Luozzo, M. Fontana, B.Arcondo. Modelling of induction heating of carbon steel tubes: Mathematical analysis, numerical simulation and validation, Journal of Alloys and Compounds. 536S (2012) S 564- S568.

[7] Z Xuebiao, C Cheng, L Yujun, et al. Numerical analysis of multi-field coupled process of steel plate bending by moveable induction heating, Journal of Harbin Engineering University. 36 (2015) 473-478.

[8] G Kai, Q Xunpeng, W Zhou, et al. Effect of magnetizer geometry on the spot induction heating process, Journal of Materials Processing Technology. 231 (2015) 125-136.

[9] M. Kranjc, A. Zupanic, D. Miklavcic, et al. Numerical analysis and thermographic investigation of induction heating, International Journal of Heat and Mass Transfer. 53 (2010) 585-3591.

[10] K. H. Cho. Coupled electro-magneto-thermal model for induction heating process of a moving billet, International Journal of Thermal Sciences. 60 (2015) 195-204.

[11] S Honghua, L Qilin, X Jiuhua, et al. Study on influence factors of temperature in localized ultrahigh frequency induction brazing, Transactions of the China Welding Institution. 33 (2012) 13-17. 